\title{
Laktat als Marker zur Risikoabschätzung geeignet
}

Um bei normotensiven Patienten das Komplikationsrisiko bei akuter Lungenembolie zu beurteilen, wurden bislang hauptsächlich kardiale Marker verwendet. Diese haben allerdings nur eine eingeschränkte Vorhersagekraft. Nun erwies sich der Plasmalaktatspiegel als aussagekräftiger Prädiktor für Verlauf und Mortalität der Erkrankung. Thorax 2015; 70: 333-338

An der prospektiven Kohortenstudie nahmen 496 Patienten mit akuter symptomatischer Lungenembolie teil. Von allen Teilnehmern wurde initial der Laktatspiegel bestimmt. Ein Wert von $\geq 2 \mathrm{mmol} / \mathrm{l}$ galt als hoch. Innerhalb von 7 Tagen nach der Diagnose wurden die mit der Lungenembolie assoziierten Komplikationen Mortalität und hämodynamischer Kollaps erfasst. Bei 20 der 496 Teilnehmer traten Komplikati- onen auf. Diese Patienten hatten höhere Laktatspiegel als Probanden ohne Komplikationen (Median 2,66 vs. 1,20 mmol/l; $\mathrm{p}<0,001)$. In der Gruppe mit hohem Laktatspiegel waren insgesamt $10,4 \%$ der Patienten von Komplikationen betroffen gegenüber $1,7 \%$ in der Gruppe mit normalem Laktat. Dies entsprach einem positiven Vorhersagewert von 10,4\% und einer negativen Vorhersagekraft von 98,3\%.
Folgende Marker waren Prädiktoren für den Verlauf einer akuten Lungenembolie:

- hoher Laktatspiegel (adjustiertes Odds

Ratio [OR 5,3]; 95\%-KI 1,9-14,4),

- rechtsventrikuläre Dysfunktion (3,6; 95\%-KI 1,4-9,6),

- Tachykardie (3,6; 95\%-KI 1,4-9,0),

- systolischer Blutdruck 90-100 mmHg (3,4; 95\%-KI 1,1-10,6).

Die Kombination von Markern für eine rechtsventrikuläre Dysfunktion und eine Myokardschädigung mit dem Laktatspiegel erhöhte deren positive Vorhersagekraft von 8,6 auf $17,9 \%$.

\section{Fazit}

In der Studie war der Laktatspiegel ein wertvoller Marker, um kurzfristige Komplikationen bei normotensiven Lungenembolie-Patienten vorherzusagen. In Kombination mit Markern für eine rechtsventrikuläre Dysfunktion und eine Myokardschädigung könnte Laktat als Richtwert für Therapieentscheidungen dienen, so die Autoren. Limitiert wird die Studie u.a. dadurch, dass die Laktatwerte nur 1-mal erhoben wurden.

Vanessa Keinert, Neckargemünd

\section{Reduce to Quit: Rauchverzicht in Raten mit Vareniclin}

Ein internationales Forscherteam um J. O. Ebbert et al. hat eine Therapie für Raucher untersucht, die ihren Zigarettenkonsum vor der Abstinenz zunächst reduzieren wollten. Die Studie hat gezeigt, dass bei intensiver Betreuung und Behandlung mit Vareniclin gute Ergebnisse erzielt werden können. JAMA 2015; 313: 687-694

Die Reduce-to-Quit-Methode unter Behandlung mit Vareniclin konnte eine deutliche Erhöhung der CAR (Continious Abstinence Rate) im Studienzeitraum erreichen. Bei diesem Ansatz wird der Zigarettenkonsum während einer ersten Behandlungsphase bei Gabe von Vareniclin und klinischer Unterstützung zunächst stufenweise reduziert. Ziel dieser Phase war es, nach 12 Wochen einen kompletten Rauchverzicht zu erreichen. Die Behandlung mit Vareniclin sollte die Teilnehmer anschließend weitere 12 Wochen bei ihrem Entzug unterstützen. Primärer Studienendpunkt der doppelblinden, randomisierten und placebokontrollierten Studie war die Kohlenmonoxid-kontrollierte CAR in den Wochen 15-24. Die 1510 Teilnehmer der Studie wurden in eine Vareniclin- und eine Placebo-Gruppe unterteilt.

Im Verumarm zeigte sich in den Therapiewochen 15-24 eine durchschnittliche CAR von 32,1\% (vs. 6,9\% unter Placebo). In den Wochen 21-24 konnte unter Behandlung mit Vareniclin eine CAR von 37,8\% verzeichnet werden. Der Placeboarm kam im gleichen Zeitraum nur auf eine durchschnittliche CAR von 12,5\%. Der Effekt der Vareniclin-Behandlung setzte sich in den Wochen 21-52 fort: In der VareniclinGruppe wurden noch durchschnittlich $27 \%$ der Raucher als abstinent gewertet (vs. 9,9\% unter Placebo). Neben typischen Effekten des Nikotinentzugs (Obstipation, Gewichtszunahme etc.) zeigten sich im Studienzeitraum häufig auftretende Fälle von Schwindel und Schlaflosigkeit. Auch kam es in der Verum-Gruppe gehäuft zu abnormen Träumen.

\section{Fazit}

Unter intensiver medizinischer Betreuung können Patienten bei ihrer Rauchentwöhnung erfolgreich mit Vareniclin unterstützt werden. Der Reduce-ToQuit-Ansatz sollte Rauchern vorgeschlagen werden, die zum Zeitpunkt der Beratung nicht zu einem sofortigen Entzug bereit sind, so die Autoren. Dabei ist es wichtig einen konkreten Termin für die vollständige Abstinenz festzulegen und im Vorfeld eine stufenweise Reduktion des Zigarettenkonsums zu erreichen.

Felix Lörch, Stuttgart 